\title{
Positive Effect on Behaviour of Autistic Children by Supplementation of New Complexed Cholecalciferol is Combined With Reduction of Lipid Peroxidation: A Pilot Study
}

\author{
Joachim Greilberger ${ }^{1,2}$, Michaela Greilberger ${ }^{2}$ and Ralf Herwig, ${ }^{3,4 *}$ \\ ${ }^{1}$ Institute for Physiological Chemistry, Medical University Graz, Austria \\ ${ }^{2}$ Institute of Scientific Laboratory, Schwarzl-Medical Center, Austria \\ ${ }^{3}$ Mens's Health Clinic, Austria \\ ${ }^{4}$ German Medical Center, United Arabic Emirates
}

Submission: May 14, 2018; Published: May 21, 2018

*Corresponding author: Ralf Herwig, Mens's Health Clinic, Vienna, German Medical Center, Dubai, Austria, Email: dr.ralf.herwig@gmail.com

\begin{abstract}
Aim: Oxidative stress may play a role in the development and clinical manifestations of autism. Children with autism are more vulnerable to oxidative stress in the form of increased lipid peroxidation. Vitamin D supplementation is known to decrease oxidative stress by ist antiinflammantary properties.

Methods: Autistic children ( $\mathrm{n}=12)$ were administerd for five weeks complexed cholecalciferol-dimer (ImmunoDß) orally twice a week. Before and after five weeks supplementation of complexed cholecalciferol plasma samples from ten autistic children were collected and lipid peroxidation markers hydroxyalkenales (HAE), malondialdehyde (MDA) and lipid peroxides were measured. Additionally, the standardized University of Cambridge AQ-10 Questionaire (AQ-10) in its original and slightly modified version to test for changes in behavior.

Results: After supplementation with complexed vitamin D HAE decreased significantly from $1.99 \pm 0.61 \mu \mathrm{M}$ to $1.09 \pm 0.65 \mu \mathrm{M}(\mathrm{p}<0.02)$ and MDA significantly from $0.99 \pm 0.48 \mu \mathrm{M}$ to $0.33 \pm 0.18 \mu \mathrm{M}(\mathrm{p}<0.01)$ in the autistic children. The content of lipid peroxides in autistic children were also reduced significantly from $235.8 \pm 136.9 \mu \mathrm{M}$ to115.2 $\pm 64.8 \mu \mathrm{M}(\mathrm{p}<0.02)$. The behavior of the probants improved significantly, which is expressed by the original and modified version of the AQ-10 ( $\mathrm{p}=0.001)$. In 6 out of 12 of the cases, an ASD diagnosis would not be further considered according to the test results. Furthermore, significant changes in various questions could be observed, demonstrating an improvement in relevant facets of social behavior.
\end{abstract}

Conclusion: Supplementation of complexed vitamin D dimer is suggested to decrease oxidative stress and inflammation in autistic children and may improve their social behavior.

Keywords: Vitamin D deficiency; Immunotherapy; Autism; ASD; ImmunoD® (VitD-dimer-complex)

\section{Introduction}

Autism and autism spectrum disorders (ASDs) are complex heterogeneous neurodevelopmental conditions [1]; they are a frequent developmental disorder, characterized by pervasive deficits in social interaction, impairment in verbal and nonverbal communication, and stereotyped patterns of interests and activities [2].

An increasing amount of evidence points to the possibility that gestational and early childhood vitamin $\mathrm{D}$ deficiency $[25(\mathrm{OH}) \mathrm{D}<40 \mathrm{ng} / \mathrm{ml}]$ cause some cases of autism [3]. The conflation of in vitro, ex vivo, and animal model data provide compelling evidence that vitamin $\mathrm{D}$ has a crucial role in proliferation, differentiation, neurotrophism, neuroprotection, neurotransmission, and neuroplasticity. Vitamin D exerts its biological function not only by influencing cellular processes directly, but also function by influencing cellular processes as well as by gene expression through vitamin $\mathrm{D}$ response elements [4].

Moreover, immune system dysregulation is well-recognized in autism and thought to be part of the etiology of this disorder [5]. The prevailing hypothesis is that some combination of immune factors including maternally-developed antibodies to fetal brain prime microglia in such a way as to preclude their normal functions of directing neuronal migration and pruning [6,7]. Endogenous glycosylated vitamin D binding protein, a macrophage activating factor, is assumed to play an important role in this immune dysregulation in autism via the 
endocannabinoid system [5] which also involves also increase of oxidative stress. It is suggested that oxidative stress itself might be one of the major causes for autism. Essa et al. [8] reported a significant increase of lipid peroxidation end products of polyunsaturated fatty acids like malondialdehyde (MDA) in autitsic children, compared to a healthy population.

We recently described a supplementation with a newly developed protein-stabilized vitamin D complex ImmunoD® (HG Pharma, Vienna, Austria) in patients with neurodegenerative diseases with a reduction in symptoms and a reduction of oxidative stress parameter [9]. The aim of this study was to evaluate the effect of ImmunoD $®$ supplementation in autistic children and to estimate lipid peroxides and their end products lipid aldehydes, like malondialdehyde (MDA) and hydroxyalkenales (HAE) before and after supplementation.

\section{Material and Methods}

\section{Patients}

Of 140 children verified to suffer from Asperger syndrome and using ImmunoD $\AA$ as a supplement twelve children were elected for an online questionnaire assessment before and after ImmunoD® supplementation. ImmunoD®, obtained from $\mathrm{HG}$ Pharma GmbH (Vienna, Austria) was applied orally twice per week one ampule dissolved with $1 \mathrm{ml}$ of water over five weeks.

Altogether, 12 children could be examined with the validated and standardized University of Cambridge AQ-10 Questionaire [10] before and after five weeks of supplementation. In the original version, only 1 point can be scored for each question. The score of 1 point for definitely or slightly agree on each of items $1,7,8$, and 10 , and a score of 1 point for definitely or slightly disagree on each of items $2,3,4,5,6$, and 9 should be given. At a cut-point of 6 on the original AQ-10 child, sensitivity for positive diagnosis of autism was 0.95 , specificity was 0.97 , and PPV was 0.94 [10].

For further calculation reasons, the AQ-10 was slightly modified in scoring. In the modified calculation version (Table 1 ), the maximum score of 40 points in the modified version is equaling 10 points of the original.

Table 1.

\begin{tabular}{|c|c|}
\hline & Questions \\
\hline 1 & S/he often notices small sounds when others do not \\
\hline 2 & S/he usually concentrates more on the whole picture, rather than the small details \\
\hline 3 & In a social group, s/he can easily keep track of several different people's conversations \\
\hline 4 & S/he finds it easy to go back and forth between different activities \\
\hline 5 & S/he doesn't know how to keep a conversation going with his/her peers \\
\hline 6 & Shen s/he is read a story, s/he finds it difficult to work out the character's intentions or feelings \\
\hline 7 & When s/he was in preschool, s/he used to enjoy playing games involving pretending with other children \\
\hline 8 & S/he finds it easy to work out what someone is thinking or feeling just by looking at their face \\
\hline 9 & \\
\hline
\end{tabular}

\section{Oxidative stress blood plasma analysis}

Additionally to the questionaire, EDTA-plasma was obtained from ten out of the twelve children before and 5 weeks after supplementation, and stored at $-70{ }^{\circ} \mathrm{C}$ till measurments. The content of lipid peroxidation end products like malondialdehyde (MDA) and hydroxyalkenales (HAE) was obtained from Oxford Biomedical Research (Oxford, USA) [11,12]. This spectrophotometrical method was designed to assay either MDA or 4-hydroxyalkenals, using a chromegenic reagent at $586 \mathrm{~nm}$.

The determination of peroxides was performed by the reaction of a peroxidase with peroxides in the sample followed by the conversion of TMB to a colored product (Immundiagnsotik AG, Bensheim, Germany). After adding a stop solution, the samples were measured at $450 \mathrm{~nm}$ in a microtiter plate reader. The quantification is performed by the delivered calibrator. $10 \mu \mathrm{L}$ of sample, calibrators, controls and blank solution were pipeted into appropriate wells of the 96 microtitration plate. After adding of $100 \mu \mathrm{L}$ reaction buffer $\mathrm{A}$, the absorbance at 450 $\mathrm{nm}$ should be read. The reaction was initiated by adding $100 \mu \mathrm{L}$ of the reaction mixture. After 15 minutes incubation at $37^{\circ} \mathrm{C}$, the reaction was stopped by adding $50 \mu$ l stop solution. Again, the absorbance was read at $450 \mathrm{~nm}$. After substraction of absorbance of the first measurement, the concentration was measured with linear regression of the standard curve of the used calibrators.

\section{Statistics}

Data were anonymized, and the results are presented as mean \pm standard deviation (SD). Statistical differences in the scores of each scale, before and after five weeks of vitamin D - ImmunoD® supplementation period, were determined by a paired t-test under the assumption of given normality of the data, re-conducted to paired t-test. Significance between groups was tested with linear regression analysis. Statistical Package for Social Sciences program (IBM Corp. Released 2016. IBM SPSS Statistics for Mac, Version 24.0. Armonk, NY, USA) version 24 was used for data analysis. Results were considered statistically significant with $\mathrm{p}$ values $\leq .05$ (Figure 1 ). 


\section{Figure 1}

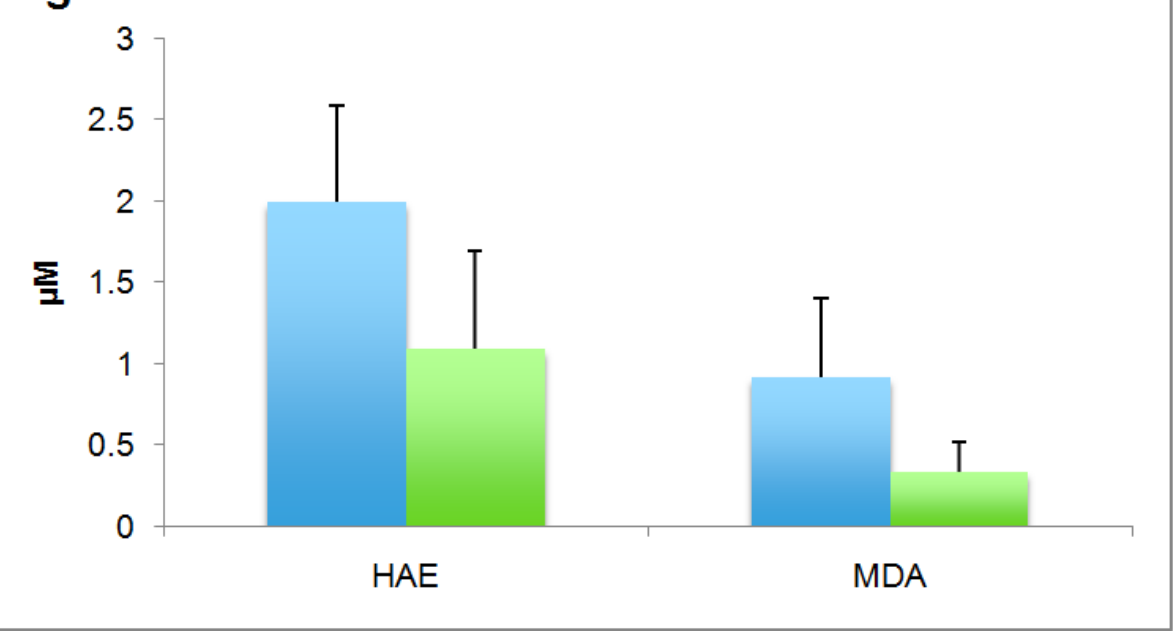

figure 3: Determination of plasma HAE and plasma MDA before (blue bars) and after 5 weeks (red bars) oral supplementation of complexed cholecalciferol in autistic children $(n=7)$.

\section{Results}

\section{AQ-10 Questionaire}

The mean age of the twelve participants was 6.5 years $( \pm$ 2.5 , minimum age 4 , maximum age 11 ). No side-effects have been noticed in any of the test persons. All twelve children would have been considered to be suffering from ASD, using the original version of the AQ-10. Interestingly, a clear decrease in mean score was noticed after ImmunoD® intake: the mean score before was 7.92 points (pts) $( \pm 1.31)$ compared to 5.50 pts $( \pm 1.78)$ after five weeks (Figure 2$)$; this result was statistically significant ( $p=0.005)$. Additionally, six out of twelve $(50 \%)$ of the participants would not further have been considered to suffer from ASD after supplementation due to a score below 6 points in the AQ-10. The modified version of the AQ-10 demonstrated a decrease in cumulative scoring from a mean of $32.33 \mathrm{pts}( \pm 4.58)$ before, to a mean of 26 pts $( \pm 2.26)$ after intake, the results are graphically illustrated in Figure 3. Although, the standard deviation is relatively high, this reduction was statistically different $(p=0.001)$. Furthermore, a statistically significant difference could be observed in question $4(p=0.002)$, question 5 $(\mathrm{p}=0.001)$, question $6(\mathrm{p}=0.039)$, question $8(\mathrm{p}=0.002)$, question $9(\mathrm{p}=0.002)$ and question $10(\mathrm{p}=0.013)$. No significant change was noted in question 1 , question 3 and question 7 , whereas a clear tendency could be seen in question $2(p=0.07)$. This non-significant result might be due to the fact, that some of the children were too young to answer the questions properly, especially in terms of concentration on pictures, tracking conversations and story reading (Table 2).

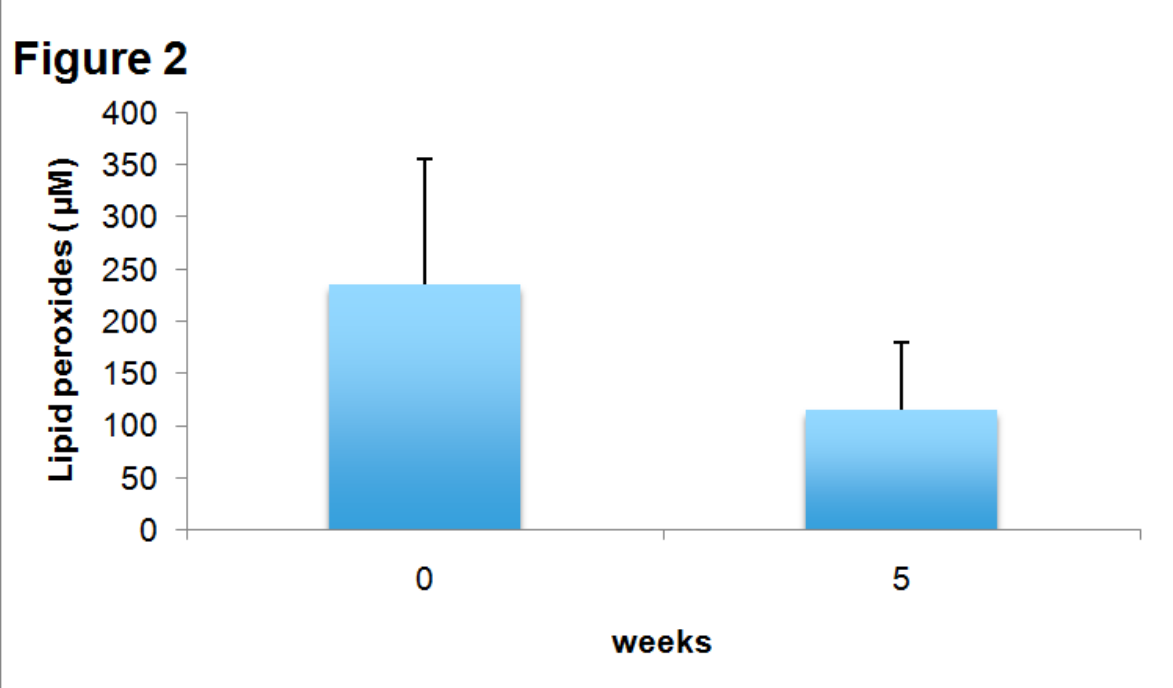

figure 4: Determination of plasma lipid peroxides before and after 5 week oral supplementation of complexed cholecalciferol in autistic children $(n=7)$. 


\section{Current Trends in Biomedical Engineering \& Biosciences}

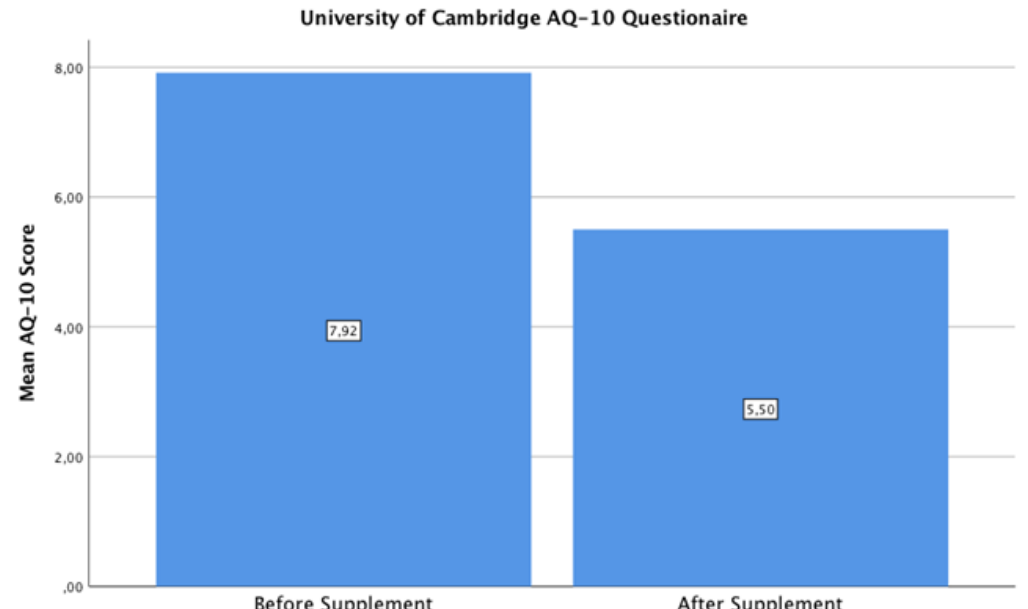

figure 7: Comparison of mean score value with standard deviation using the Asperger Syndrome Questionaire (AQ) Short-Form before and after 5 weeks of ImmunoD® supplementation.

Table 2.

\begin{tabular}{|c|c|c|c|c|}
\hline Question & Definitely Agree & Slightly Agree & Slightly Disagree & Definitely Disagree \\
\hline 1 & 4 & 3 & 2 & 1 \\
\hline 2 & 1 & 2 & 3 & 4 \\
\hline 3 & 1 & 2 & 3 & 4 \\
\hline 4 & 1 & 2 & 3 & 4 \\
\hline 5 & 1 & 2 & 3 & 4 \\
\hline 6 & 1 & 2 & 2 & 1 \\
\hline 7 & 4 & 3 & 3 & 4 \\
\hline 9 & 4 & 3 & 3 & \\
\hline
\end{tabular}

\section{Oxidative stress measurements}

Figure 3 shows the concentration of hydroxyalkenale (HAE) and malondialdehyde (MDA) before and after 5 weeks of oral supplementation of complexed cholecalciferol in human plasma of autistic children. HAE decreased significantly from $1.99 \pm 0.61 \mu \mathrm{M}$ to $1.09 \pm 0.65 \mu \mathrm{M}(\mathrm{p}<0.02)$, MDA significantly from $0.99 \pm 0.48 \mu \mathrm{M}$ to $0.33 \pm 0.18 \mu \mathrm{M}(\mathrm{p}<0.01)$. Lipid peroxides, a product of lipid aldehydes, was increased in autistic children with $235.8 \pm 136.9 \mu \mathrm{M}$, compared to normal values in healthy grownups figure 4 [9]. After complexed vitamin D supplementation, the amount of lipid peroxides, too, was significant lower $(115.2 \pm 64.8 \mu \mathrm{M})$ compared to the amount before supplementation $(\mathrm{p}<0.02)$.

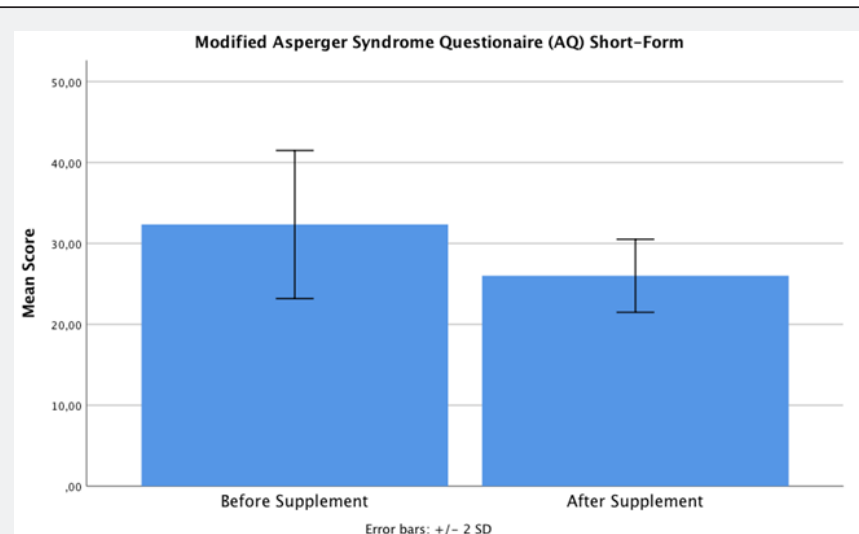

figure 2: Comparison of mean score value with standard deviation using the modified Asperger Syndrome Questionaire (AQ) Short-Form before and after 5 weeks of $I m m u n o D \otimes$ supplementation. 


\section{Discussion}

Various roles have been attributed to vitamin D as to the pathogenesis and prevention of several diseases such as cancer, cardiovascular disease, multiple sclerosis, diabetes, autism and autoimmune diseases [13]. Autism or autistic spectrum disorder (ASD) comprises a wide range of neurodevelopmental disorders characterized by problems with social skills, repetitive behaviors, speech and nonverbal communication, as well as by unique abilities. According to Diagnostic and Statistical Manual of Mental Disorders version 5 (DSM-5), ASD includes autistic disorder, childhood disintegrative disorder, pervasive developmental disorder-not otherwise specified (PDD-NOS) and Asperger's syndrome [14]. Studies have demonstrated that vitamin D deficiency during pregnancy and in individuals increased the risk of ASD [15].

Furthermore, children with autism have more vitamin D deficiencies than control children, and vitamin D co-deficiency may exacerbate the symptoms of children with ASD [16]. Therefore, Cannell et al. [17] stated, that as the American Academy of Pediatrics recommends vitamin D supplementation during infancy and childhood, pediatricians and family practitioners should evaluate the current evidence on autism and vitamin D and act accordingly.

Also, it is evident, that frontline health professionals need a "red flag" tool, to aid their decision making about whether to make a referral for a full diagnostic assessment for an autism spectrum condition (ASC) in children and adults [10].

At a cut-off point of 6 on the AQ-10 child demonstrates a sensitivity of 0.95 with a specificity of 0.97 , and a positive predictive value of 0.94 . These results can consider this questionnaire to be a reliable tool in primary diagnostics of ASD [10].

We have used the AQ-10 child, as well as a calculatory modified version, to compare the ASD situation before and after intake of a complexed Vitamin D supplementation, and to demonstrate a significance in behavior change according to the implemented questions. We found a dramatic reduction in conspicuous behavior, reflected by a significant decrease in scoring could be observed, using the AQ- 10 child in the original and modified versions. In $50 \%$ of the cases the children would not have been considered of being suffering from ASD anymore after supplementation with ImmunoD $囚$. A cut-off for possible ASD diagnosis was not calculated in the modified version due to the low number of participants.

It is evident that oxidative stress is involved in the primary etiological mechanism of ASD. This is in line with El-Anasary et al.'s report that the usage of ROC curves of several oxidative stress markers combined with impaired energy metabolism markers are suitable in the identification of this etiological mechanism of ASD [18]. A further study [8] described a significant increase of lipid peroxides and lipid aldehydes in urine from autistic children compared to a healthy age matched control group. We observed also a significant increase of lipid peroxides and lipid aldehydes (MDA and HAE) in plasma of autistic children, which echoes the study results of Essa et al. [8]. After supplementation with the dimeric complexed vitamin $\mathrm{D}$, the content of lipid peroxides and lipid aldehydes decreased within five weeks, which reflects a decrease in oxidative stress and inflammation.

On the basis of the results obtained and reported above, we suggest to interpret our observations in terms of a strong relation between oxidative stress and behavior in autistic children. This can be reduced by intake of anti-oxidative supplements like the afore mentioned ImmunoD®. These findings should lead to further scrutination of oxidative stress involvement and its possible modification via Vitamin D supplementation in ASD. As this is a preliminary report, the study is lacking of a higher number of probands and further studies with larger population of test persons have to be performed to verify these preliminary findings.

\section{References}

1. Diagnostic APA (2000) Diagnostic and Statistical Manual of Mental Disorders, Text Revision. American Psychiatric Association.

2. Saad K, Abdel-Rahman AA, Elserogy YM, Al-Atram AA, El-Houfey AA (2018) Randomized controlled trial of vitamin D supplementation in children with autism spectrum disorder. J Child Psychol Psychiatry 59: 20-29.

3. Cannell JJ (2017) Vitamin D and autism, what's new. Rev Endocr Metab Disord 18: 183-193

4. DeLuca GC, Kimball SM, Kolasinski J, Ramagopalan SV, Ebers GC (2013) Review: the role of vitamin D in nervous system health and disease. Neuropathol Appl Neurobiol 39: 458-484.

5. Siniscalco D, Bradstreet JJ, Cirillo A, Antonucci N (2014) The in vitro GcMAF effects on endocannabinoid system transcriptionomics, receptor formation, and cell activity of autism-derived macrophages. J Neuroinflammation 11: 78.

6. Bauman MD, Iosif AM, Ashwood P, Braunschweig D, Lee A, et al. (2013) Maternal antibodies from mothers of children with autism alter brain growth and social behavior development in the rhesus monkey. Transl Psychiatry 3: e278.

7. Gesundheit B, Rosenzweig JP, Naor D, Lerer B, Zachor DA, et al. (2013) Immunological and autoimmune considerations of Autism Spectrum Disorders. J Autoimmun 44: 1-7.

8. Essa MM, Guillemin GJ, Waly MI, Al-Sharbati MM, Al-Farsi YM, et al. (2012) Increased markers of oxidative stress in autistic children of the Sultanate of Oman. Biol Trace Elem Res 147: 25-27.

9. Greilberger J, Greilberger M, Herwig R (2017) Measurement of oxidative stress parameters, vitamin $\mathrm{D}$ and vitamin $\mathrm{D}$ binding protein during vitamin $\mathrm{D}$ treatment in a patient with amyotrophic lateral sclerosis. Integr Mol Med 4: 1-5.

10. Allison C, Auyeung B, Baron-Cohen S (2012) Toward brief "Red Flags" for autism screening: The Short Autism Spectrum Quotient and the Short Quantitative Checklist for Autism in toddlers in 1,000 cases and 3,000 controls [corrected]. J Am Acad Child Adolesc Psychiatry 51: 202-212.e7.

11. Lamprecht M, Bogner S, Schippinger G, Steinbauer K, Fankhauser F, et al. (2012) Probiotic supplementation affects markers of intestinal barrier, oxidation, and inflammation in trained men; a randomized, double-blinded, placebo-controlled trial. J Int Soc Sports Nutr 9: 45. 
12. Esterbauer H, Schaur RJ, Zollner H (1991) Chemistry and biochemistry of 4-hydroxynonenal, malonaldehyde and related aldehydes. Free Radic Biol Med 11: 81-128.

13. Delvin E, Souberbielle JC, Viard JP, Salle B (2014) Role of vitamin D in acquired immune and autoimmune diseases. Crit Rev Clin Lab Sci 51 232-247.

14. Máčová L, Bičíková M, Ostatníková D, Hill M, Stárka L (2017) Vitamin D, neurosteroids and autism. Physiol Res 66: S333-S340.

15. Jia F, Shan L, Wang B, Li H, Miao C, et al. (2017) Bench to bedside review: Possible role of vitamin D in autism spectrum disorder. Psychiatry Res 260: 360-365.

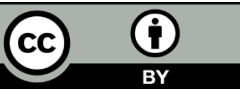

This work is licensed under Creative Commons Attribution 4.0 License

DOI: $10.19080 / C T B E B .2018 .14 .555893$
16. Guo M, Zhu J, Yang T, Lai X, Lei Y, et al. (2018) Vitamin A and vitamin $\mathrm{D}$ deficiencies exacerbate symptoms in children with autism spectrum disorders. Nutr Neurosci 16: 1-11.

17. Cannell JJ (2017) Vitamin D and autism, what's new. Rev Endocr Metab Disord 18: 183-193.

18. El-Ansary A, Al-Daihan S, Al-Dbass A, Al-Ayadhi L (2010) Measurement of selected ions related to oxidative stress and energy metabolism in Saudi autistic children. Clin Biochem 43: 63-70.

Your next submission with Juniper Publishers
will reach you the below assets
- Quality Editorial service
- Swift Peer Review
- Reprints availability
- E-prints Service
- Manuscript Podcast for convenient understanding
- Global attainment for your research
- Manuscript accessibility in different formats
( Pdf, E-pub, Full Text, Audio)
- Unceasing customer service
Track the below URL for one-step submission
https://juniperpublishers.com/online-submission.php

